\title{
Validação de aplicativos no contexto da saúde: revisão integrativa
}

\author{
Application validation in the health context: integrative review \\ Validación de aplicaciones en el contexto de la salud: revisión integradora
}

Eliany Nazaré Oliveira

ORCID: https://orcid.org/0000-0002-6408-7243

Universidade Estadual Vale do Acaraú, Brasil

E-mail: elianyy@hotmail.com

Bruna Torres Melo

ORCID: https://orcid.org/0000-0001-8815-6971

Universidade Estadual vale do Acaraú, Brasil

E-mail: brunaa8@hotmail.com

Andressa Galdino Carvalho

ORCID: https://orcid.org/0000-0001-8952-3366

Universidade Estadual vale do Acaraú, Brasil

E-mail:andressacar22@hotmail.com

Francisca Verônica Dias Melo

ORCID: https://orcid.org/0000-0002-4794-7520

Universidade Estadual Vale do Acaraú, Brasil

E-mail: veronica.dias626@gmail.com

João Breno Cavalcante Costa

ORCID: https://orcid.org/0000-0002-4512-1944

Universidade Federal do Ceará, Brasil

E-mail: brenocavalcanteenfermagem@gmail.com

Gleisson Ferreira Lima

ORCID: https://orcid.org/0000-0002-5465-2675

Escola de Saúde Pública Visconde de Saboia, Brasil

E-mail: gleisson_nega@hotmail.com

Heliandra Linhares Aragão

ORCID: https://orcid.org/0000-0001-6881-7250

Universidade Estadual Vale do Acaraú, Brasil

heliandrabj@ hotmail.com

Flávio Araújo Prado

ORCID: https://orcid.org/0000-0002-8310-2330 Prefeitura Municipal de Forquilha, Brasil

E-mail: sesa.forquilha@gmail.com

Lucas Marques Ribeiro

ORCID: https://orcid.org/0000-0003-4955-0815

Universidade Estadual Vale do Acaraú, Brasil E-mail: lucasribeiro1024b@gmail.com

Maria de Lourdes Bezerra da Silva

ORCID: https://orcid.org/0000-0002-2952-0996

Universidade Estadual Vale do Acaraú, Brasil E-mail: lourdes_celio@yahoo.com.br

Ludmilla Alves Santos

ORCID: https://orcid.org/0000-0003-4592-527X

Prefeitura Municipal de Forquilha, Brasil

E-mail: ludmillaalves91@hotmail.com

\begin{abstract}
Resumo
A tecnologia da informação e comunicação compreende um conjunto de recursos tecnológicos que viabilizam o processo de comunicação. Dentre as funções disponíveis, cita-se o uso de aplicativos, utilizados em diversas áreas e que podem abranger diferentes temáticas. No âmbito da saúde móvel, têm participação na promoção e educação em saúde, caracterizando-se como ferramenta importante para a população. O objetivo do presente estudo foi identificar, nas publicações nacionais e internacionais, indexadas nas bases de dados, os principais métodos adotados pelos pesquisadores para validação de aplicativos na área da saúde. Trata-se de um estudo de revisão integrativa em que se utilizou da seguinte busca: "Mobile Applications" AND "Health" AND "Validation Study". Totalizaram-se seis pesquisas, redigidas em língua inglesa, predominando, entre estas, o intuito de examinar a validade e confiabilidade do aplicativo analisado (A3 e A5) e validar o aplicativo (A4 e A6). Os achados demonstraram bom desempenho dos aplicativos. A avaliação de aplicativos móveis, por meio da validação, garante mais segurança à tecnologia,
\end{abstract}


demonstrada pela eficácia. Com isso, destaca-se a necessidade do processo de validação com rigor metodológico, assim como publicações acerca dessa temática voltadas ao âmbito da saúde.

Palavras-chave: Aplicativos móveis; Estudo de validação; Atenção à saúde.

\begin{abstract}
Information and communication technology comprises a set of technological resources that enable the communication process. Among the available functions, the use of applications is cited, used in different areas, and covering different themes. In the context of mHealth, they participate in health promotion and education, characterized as an important tool for the population. The aim of this study was to identify, in national and international publications indexed in databases, the main methods adopted by researchers to validate applications in the health area. This is an integrative review study in which the following search was used: "Mobile Applications" AND "Health" AND "Validation Study". Six studies were totaled, written in English, predominating, among them, the intention to examine the validity and reliability of the analyzed application (A3 and A5) and to validate the application (A4 and A6). The findings demonstrated a good application performance. The evaluation of mobile applications, through validation, guarantees more security to the technology, demonstrated by its effectiveness. Given the above, the need for a validation process with methodological rigor is highlighted, as well as publications on this topic aimed at the health field.
\end{abstract}

Keywords: Mobile applications; Validation study; Delivery of health care.

\title{
Resumen
}

La tecnología de información y comunicación comprende un conjunto de recursos tecnológicos que posibilitan el proceso de comunicación. Entre las funciones disponibles, se cita el uso de aplicaciones, utilizadas en diferentes áreas y que cubren diferentes temas. En el contexto de la mHealth, participan en la promoción y educación de la salud, caracterizada como una herramienta importante para la población. El objetivo de este estudio fue identificar, en publicaciones nacionales e internacionales indexadas en bases de datos, los principales métodos adoptados por investigadores para validar aplicaciones en el área de la salud. Se trata de un estudio de revisión integradora en el que se utilizó la siguiente búsqueda: "Mobile Applications" AND "Health" AND "Validation Study". Se totalizaron seis estudios, redactados en inglés, predominando, entre ellos, la intención de examinar la validez y fiabilidad de la aplicación analizada (A3 y A5) y de validar la aplicación (A4 y A6). Los resultados demostraron un buen rendimiento de la aplicación. La evaluación de aplicaciones móviles, mediante validación, garantiza más seguridad a la tecnología, demostrada por su efectividad. Dado lo anterior, se destaca la necesidad de un proceso de validación con rigor metodológico, así como publicaciones sobre este tema dirigidas al campo de la salud.

Palabras clave: Aplicaciones móviles; Estudio de validación; Atención a la salud.

\section{Introdução}

A Tecnologia da Informação e Comunicação (TIC) compreende um conjunto de recursos tecnológicos que viabilizam a comunicação nos processos existentes em diversos âmbitos. Essa tecnologia torna possível, aos profissionais, novos meios de obter resultados com maior agilidade e eficiência, possibilitando maior alcance, assim como benefícios para os pacientes (Tibes et al., 2017).

À vista disso, observa-se o crescente uso de equipamentos móveis pela população mundial, em que a utilização de smartphones tem previsão de crescimento de 13,9\% no primeiro trimestre de 2021 e 5,5\% para o ano de 2021 (International Data Corporation, 2021). No Brasil, o acesso à internet foi verificado em 79,1\% dos domicílios no ano de 2018, e a conexão, por meio do telefone móvel, é observada de forma progressiva no cotidiano dos indivíduos, sendo, na atualidade, a principal via de acesso à internet no país (Instituto Brasileiro de Geografia e Estatística, 2017).

Dentre as funções disponíveis nos smartphones, cita-se o uso de aplicativos, os quais configuram-se como softwares elaborados para instalação em dispositivos móveis (Pressman \& Maxim, 2016; Veiga et al., 2017), são utilizados em diversas áreas e podem abranger diferentes temáticas, sendo que, no âmbito da saúde móvel, ou mHealth, têm participação na promoção e educação em saúde, caracterizando-se como ferramenta importante para população (Oliveira et al., 2020)

Contudo, a despeito das grandes potencialidades, da versatilidade e de, atualmente, os aplicativos móveis se encontrarem inseridos no âmbito da saúde e disponíveis para os usuários, o simples fato de incluir TIC na prática profissional não assegura desempenho eficaz e efetivo, uma vez que as informações fornecidas e o objetivo podem ser questionáveis (Baig 
et al., 2019). Nesta perspectiva, faz-se necessária a avaliação do desempenho dessas tecnologias e a análise dos impactos ocasionados por esse tipo de ferramenta.

Desse modo, objetivou-se identificar, nas publicações nacionais e internacionais, indexadas nas bases de dados, os principais métodos adotados por pesquisadores para validação de aplicativos na área da saúde.

\section{Metodologia}

Trata-se de estudo de revisão integrativa, tipologia que se destaca dentre os demais perfis de revisão pela abordagem metodológica ampla que viabiliza a completa compreensão do fenômeno avaliado, mediante a inclusão de estudos experimentais e não experimentais (Souza et al., 2010).

Para o delineamento do estudo, percorreram-se os seguintes passos: identificação das questões de pesquisa e elaboração da questão norteadora; busca na literatura e seleção dos artigos primários; extração de dados dos estudos primários; avaliação crítica dos estudos; e síntese dos resultados (Hopia et al., 2016). Assim, a questão norteadora de pesquisa compreendeu a pergunta: quais evidências científicas estão sendo produzidas sobre a validação de aplicativos na área da saúde?

A busca por estudos, por sua vez, ocorreu no intervalo compreendido entre os dias 10 e 14 de maio do ano de 2021 e abrangeu a biblioteca Scientific Electronic Library Online (SciELO) e as bases de dados Literatura Latino-Americana e do Caribe em Ciências da Saúde (LILACS), Base de Dados de Enfermagem (BDENF), Medical Literature Analysis and Retrieval System Online (MEDLINE), Web of Science e Scopus, em que as duas primeiras bases (LILACS e BDENF) foram acessadas por meio da Biblioteca Virtual de Saúde (BVS), a seguinte (MEDLINE) pela U. S. National Library of Medicine (PUBMED) e Web of Science e Scopus, via portal CAPES.

Ainda nessa perspectiva, realizou-se a busca por termos no site dos Descritores em Ciências da Saúde (DeCS), em que os descritores encontrados foram selecionados no idioma inglês e combinados com o operador booleano AND, do seguinte modo: "Mobile Applications" AND Health AND "Validation Study". Posteriormente, aplicou-se, nos resultados obtidos, o filtro "texto completo grátis", com intuito de que, junto à escolha das palavras de busca no idioma inglês, fosse encontrado o maior número possível de estudos.

A seleção dos artigos foi realizada com base na metodologia Preferred Reporting Items for Systematic Reviews and Meta Analyses (PRISMA), de acordo com Page et al. (2021), seguindo os passos de identificação, seleção, elegibilidade e inclusão. Posterior a isso, para construir amostra ampla e robusta cientificamente, realizou-se a classificação por nível de evidência científica, observando o método proposto por Melnyk \& Fineout-Overholt (2015). Assim, procedeu-se à estratificação dos estudos em Nível I (metanálise, estudos controlados ou randomizados), Nível II (estudos experimentais), Nível III (estudos quase experimentais), Nível IV (estudos descritivos, não experimentais ou qualitativos), Nível V (relatos de experiência) ou Nível VI (consensos e opinião de especialistas).

Como critérios para inclusão no estudo, estabeleceram-se artigos originais disponíveis na íntegra e com enquadramento em níveis I, II ou III em evidência científica, sem delimitação de tempo e idioma. Os critérios de exclusão foram: duplicidade dos artigos, temas que não abordassem diretamente o assunto e dados da literatura cinzenta.

Para análise dos dados, elaborou-se tabela no Google Docs, visando categorizar os artigos, os quais foram classificados, indicando as seguintes informações: título do artigo, autores e ano de publicação, idioma, objetivo e principais resultados.

Por fim, o estudo obedeceu às disposições acerca do uso de dados disponibilizados para domínio público, estabelecidas pela Resolução 510/2016 do Conselho Nacional de Saúde (CNS). 


\section{Resultados}

Com a realização da busca, seguindo os passos detalhados, encontraram-se 164 artigos, destes, excluíram-se um por ser repetido, 107 por fuga da temática e 21 por não atendimento à questão norteadora, de modo que foram 35 os estudos selecionados. No entanto, com a estratificação desses por nível de evidência científica, removeram-se 29 por apresentação de nível IV, permanecendo, neste sentido, para estudo neste trabalho, seis pesquisas, conforme Figura 1.

Figura 1. Fluxograma de busca e seleção de artigos, segundo PRISMA (Araújo, 2020).

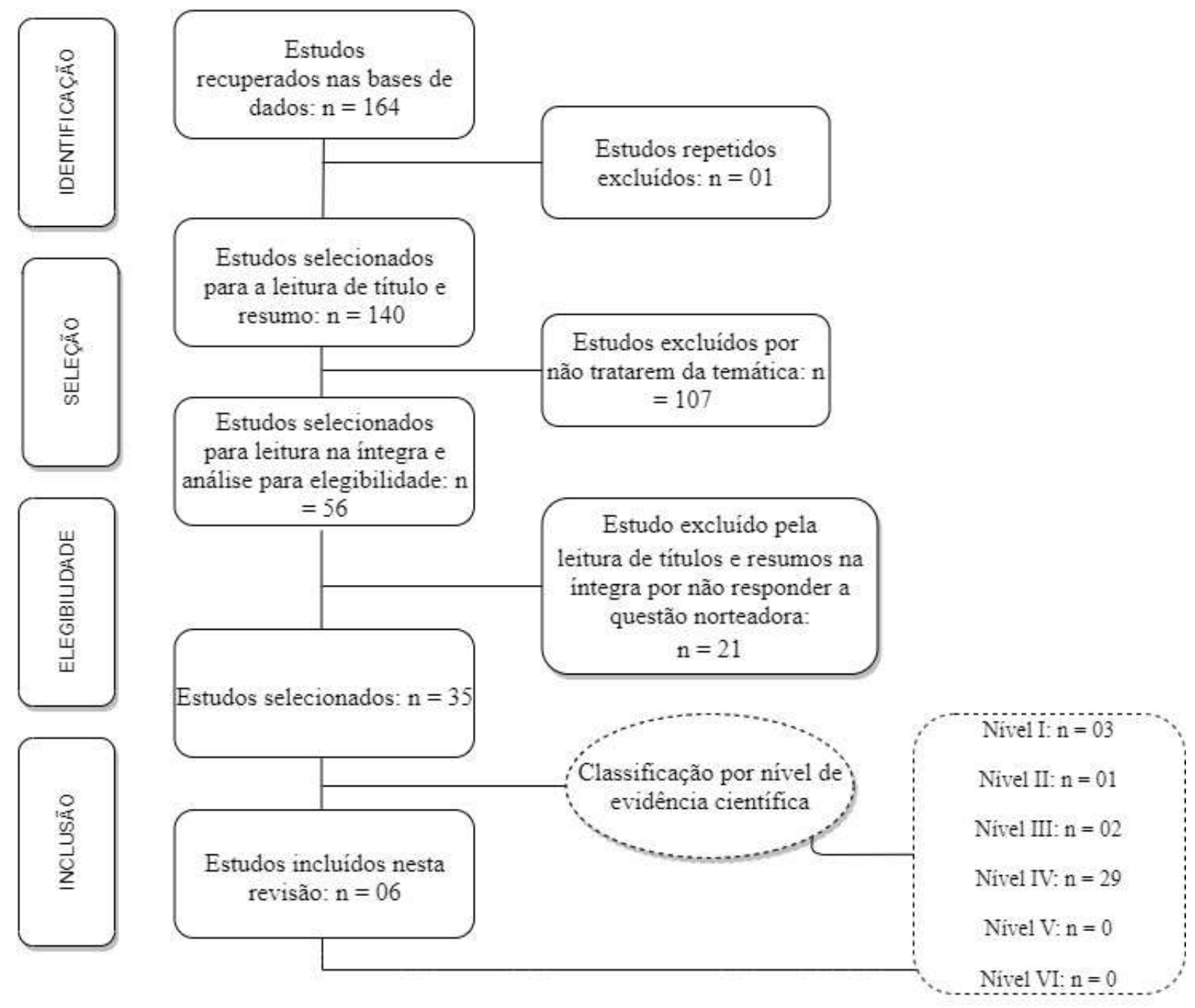

Etapa incluída pelos autores. Fonte: Autores.

A análise dos estudos evidenciou que os estudos foram redigidos em língua inglesa, recorrente o nível I de evidência científica (A3, A4 e A6) e 2017 como o ano de maior publicação (A1 e A6). Quanto à finalidade, predominou, entre as pesquisas, o objetivo de examinar a validade e confiabilidade do aplicativo analisado (A3 e A5) e validar o aplicativo (A4 e A6), ao passo que, em todas, os resultados demonstraram bom desempenho dos aplicativos. O Quadro 1 foi estruturado para sistematizar e ordenar as informações dos artigos. 
Quadro 1. Apresentação quanto ao título, autor e ano de publicação, idioma, objetivo, principais resultados e nível de evidência científica.

\begin{tabular}{|c|c|c|c|c|c|c|}
\hline Artigos & Títulos & Autores (ano) & Idioma & Objetivos & Principais resultados & NEC* \\
\hline A1 & $\begin{array}{l}\text { Educating parents about } \\
\text { their children's } \\
\text { vaccination status: a } \\
\text { user-centric mobile app }\end{array}$ & $\begin{array}{l}\text { Seeber et al. } \\
(2017)\end{array}$ & Inglês & $\begin{array}{l}\text { Estudar a capacidade } \\
\text { dos pais de } \\
\text { compreender } \\
\text { conteúdo de um } \\
\text { registro de vacinação } \\
\text { padrão com ajuda de } \\
\text { um aplicativo móvel. }\end{array}$ & $\begin{array}{l}\text { Apenas } 9 \% \text { do Grupo Recall foram } \\
\text { capazes de fornecer status de } \\
\text { vacinação completo; em média, } 39 \% \\
\text { das questões foram respondidas } \\
\text { corretamente. Usar o WHO-ICV } \\
\text { com a ajuda do VAccApp resultou } \\
\text { em } 62 \% \text { dos pais fornecendo estado } \\
\text { de vacinação completo; em média, } \\
95 \% \text { das questões foram respondidas } \\
\text { corretamente. Depois de usar o } \\
\text { VAccApp, os pais eram mais } \\
\text { propensos a se lembrar de aspectos } \\
\text { importantes da história de } \\
\text { vacinação. }\end{array}$ & III \\
\hline A2 & $\begin{array}{l}\text { Development and } \\
\text { validation of a } \\
\text { smartphone-based visual } \\
\text { acuity test (Peek Acuity) } \\
\text { for clinical practice and } \\
\text { community-based } \\
\text { fieldwork }\end{array}$ & $\begin{array}{l}\text { Bastawrous et } \\
\text { al. (2015) }\end{array}$ & Inglês & $\begin{array}{l}\text { Projetar e validar um } \\
\text { teste de acuidade } \\
\text { visual baseado em } \\
\text { smartphone que não } \\
\text { dependa rada } \\
\text { familiaridade com } \\
\text { símbolos ou letras } \\
\text { comumente usados no } \\
\text { idioma inglês. }\end{array}$ & $\begin{array}{l}\text { Os limites de IC de } 95 \% \text { para } \\
\text { variabilidade teste-reteste dos dados } \\
\text { de acuidade do smartphone foram } \pm \\
0,029 \text { logMAR. As diferenças } \\
\text { médias entre o teste baseado em } \\
\text { smartphone e o gráfico ETDRS e o } \\
\text { teste baseado em smartphone e } \\
\text { dados de acuidade Snellen foram } \\
0,07 \text { (IC 95\%, 0,05-0,09) e 0,08 (IC } \\
95 \% \text {, 0,06-0,10) logMAR, } \\
\text { respectivamente, indicando que as } \\
\text { acuidades de teste baseadas em } \\
\text { smartphone concordam bem com as } \\
\text { dos gráficos ETDRS e Snellen. Os } \\
\text { profissionais de saúde da } \\
\text { comunidade queniana local } \\
\text { aceitaram prontamente o teste do } \\
\text { smartphone Peek Acuity; exigiu } \\
\text { treinamento mínimo e não demorou } \\
\text { mais do que o teste de Snellen; (IC } \\
95 \% \text {, respectivamente; } P=0,13 \text { ). }\end{array}$ & II \\
\hline A3 & $\begin{array}{l}\text { Validation } \\
\text { reliability of and } \\
\text { smartphone application } \\
\text { for the international } \\
\text { prostate symptom } \\
\text { scoring questionnaire: a } \\
\text { cross-sectional study of } \\
\text { repeated randomized } \\
\text { measures. }\end{array}$ & $\underset{(2014)}{\text { Kim et al. }}$ & Inglês & $\begin{array}{l}\text { Examinar a validade e } \\
\text { a confiabilidade de } \\
\text { uma versão de } \\
\text { aplicativo do IPSS } \\
\text { para smartphone, por } \\
\text { meio de análise } \\
\text { quantitativa e mostrar } \\
\text { o índice de satisfação } \\
\text { em comparação com a } \\
\text { versão em papel } \\
\text { convencional do IPSS. }\end{array}$ & $\begin{array}{l}\text { A pontuação total do IPSS }(P= \\
.289) \text { e cada item do IPSS }(P=.157- \\
1.000) \text { não mostraram diferenças } \\
\text { entre a versão em papel e a versão } \\
\text { para smartphone do IPSS. Os grupos } \\
\text { LUTS leve, moderado e grave não } \\
\text { apresentaram diferenças entre as } \\
\text { duas versões do IPSS. Uma } \\
\text { correlação significativa foi } \\
\text { observada no grupo total (ICC = } \\
0,935, P<0,001) \text {. Os grupos LUTS } \\
\text { leve, moderado e grave também } \\
\text { demonstraram correlações } \\
\text { significativas (ICC }=0,616,0,549 \text { e } \\
0,548, \text { respectivamente, todos } P \\
<0,001) \text {. }\end{array}$ & I \\
\hline A4 & $\begin{array}{l}\text { Sensory-Discriminative } \\
\text { Three-Dimensional } \\
\text { Body Pain Mobile App } \\
\text { Measures } \quad \text { Versus } \\
\text { Traditional Pain } \\
\text { Measurement With a } \\
\text { Visual Analog Scale: }\end{array}$ & $\begin{array}{l}\text { Kaciroti et al. } \\
(2020)\end{array}$ & Inglês & $\begin{array}{l}\text { Validar } \\
\text { especificamente as } \\
\text { medições sensorial- } \\
\text { discriminativas } \\
\text { GeoPain de ensaio de } \\
\text { neuromodulação TMD } \\
\text { e compreender melhor }\end{array}$ & $\begin{array}{l}\text { GeoPain (PAINS) } \begin{array}{r}\text { mostrou } \\
\text { confiabilidade }\end{array} \text { teste-reteste } \\
\text { consistente em comparação com } \\
\text { VAS, demonstrada por correlações } \\
\text { lag-auto mais altas em todos os } \\
\text { dados do ensaio clínico. }\end{array}$ & I \\
\hline
\end{tabular}




\begin{tabular}{|c|c|c|c|c|c|c|}
\hline & Validation Study & & & $\begin{array}{l}\text { a confiabilidade para } \\
\text { avaliar o impacto da } \\
\text { dor sensorial e o } \\
\text { tamanho da amostra de } \\
\text { pacientes necessários } \\
\text { em comparação com o } \\
\text { escore VAS } \\
\text { tradicional. }\end{array}$ & & \\
\hline A5 & $\begin{array}{l}\text { Validating the use of a } \\
\text { smartphone app for } \\
\text { remote administration of } \\
\text { a fear conditioning } \\
\text { paradigm }\end{array}$ & $\begin{array}{l}\text { Purves et al. } \\
(2019)\end{array}$ & Inglês & $\begin{array}{lr}\text { Examinar } & \text { a } \\
\text { confiabilidade } & \text { a } \\
\text { validade desta } & \text { nova } \\
\text { abordagem } & \text { de } \\
\text { aplicativo. } & \end{array}$ & $\begin{array}{l}\text { As diferenças individuais nos } \\
\text { resultados do condicionamento do } \\
\text { medo estavam associadas à } \\
\text { ansiedade, fornecendo validade de } \\
\text { construto, na medida em que a tarefa } \\
\text { mostra relevância para o transtorno } \\
\text { de ansiedade. Além disso, ao } \\
\text { identificar aqueles que têm maior } \\
\text { ansiedade geral, a tarefa do } \\
\text { aplicativo demonstra algum nível de } \\
\text { validade diagnóstica. }\end{array}$ & III \\
\hline A6 & $\begin{array}{l}\text { Validation of a Tablet } \\
\text { Application for } \\
\text { Assessing Dietary } \\
\text { Intakes Compared with } \\
\text { the Measured Food } \\
\text { Intake/Food Waste } \\
\text { Method in Military } \\
\text { Personnel Consuming } \\
\text { Field Rations }\end{array}$ & $\begin{array}{l}\text { Ahmed et al. } \\
(2017)\end{array}$ & Inglês & $\begin{array}{l}\text { Validar a utilização do } \\
\text { aplicativo móvel } \\
\text { MyFitnessPal. }\end{array}$ & $\begin{array}{l}\text { Os gráficos de Bland-Altman de } \\
\text { medidas repetidas indicaram boa } \\
\text { concordância para ambos os } \\
\text { métodos (aplicativo para tablet e } \\
\text { wFR) com o método medido de } \\
\text { ingestão / desperdício de alimentos. }\end{array}$ & I \\
\hline
\end{tabular}

*NEC: Nível de Evidência Científica. Fonte: Autores.

Com relação aos aplicativos avaliados pelos estudos, atestou-se que os apps desenvolvidos, em grande parte, tiveram uso destinado a profissionais de saúde (A2, A5 e A6) e ao ambiente clínico (A2, A3, A5 e A6). Um, entretanto, diferenciou-se pela abordagem voltada ao binômio profissional de saúde-paciente (A3).

Por último, no quesito validação, os pesquisadores, majoritariamente, valeram-se da comparação de ferramentas com instrumentos validados, diferenciando-se, apenas, quanto ao método específico utilizado para esse fim: um fez uso do teste de Bland-Altman (A6), enquanto três, ou seja, a maior parte, seguiram a metodologia teste-reteste (A1, A2 e A4).

O resumo, contendo os dados referentes aos aplicativos desenvolvidos pelos estudos avaliados, encontra-se no Quadro 2.

Quadro 2. Descrição de público-alvo, finalidade e método de validação contemplados pelos aplicativos.

\begin{tabular}{|c|l|c|l|}
\hline Artigos & \multicolumn{1}{|c|}{ Público-alvo do aplicativo } & $\begin{array}{c}\text { Finalidades do } \\
\text { aplicativo }\end{array}$ & Métodos de validação do aplicativo \\
\hline A1 & Pais & Educação & $\begin{array}{l}\text { Comparação com um método validado (Teste-reteste) e a } \\
\text { Escala de Usabilidade do Sistema. }\end{array}$ \\
\hline A2 & Profissionais de saúde & Clínica & Comparação com método validado (teste-reteste). \\
\hline A3 & Profissionais de saúde e pacientes & Clínica & Coeficientes de correlação intraclasse (ICC). \\
\hline A4 & Pesquisadores e população & Pesquisa & Comparação com método validado (teste- reteste). \\
\hline A5 & Profissionais de saúde & Clínica & Coeficientes de correlação intraclasse (ICC). \\
\hline A6 & Profissionais de saúde & Clínica & $\begin{array}{l}\text { Comparação com método validado (Teste de Bland- } \\
\text { Altman). }\end{array}$ \\
\hline
\end{tabular}




\section{Discussão}

As TIC compreendem ferramentas de ampla versatilidade e utilização que, no atual contexto da humanidade, têm ganhado muito espaço, sobretudo, sob a forma de aplicativos móveis, em diversas áreas, a exemplo da saúde. Assim, considerando essa realidade, bem como a natureza delicada inerente às questões relacionadas à saúde, este trabalho se destinou a, mediante a revisão de estudos, avaliar os passos metodológicos percorridos pelos pesquisadores para validar os apps desenvolvidos no âmbito da saúde, de modo a fornecer à comunidade geral devolutiva quanto ao caráter de validade dessas tecnologias na referida área.

Nessa perspectiva, atesta-se, primeiramente, que, apesar de a saúde e os assuntos a ela atrelados serem comuns a todos os países, o inglês foi encontrado como idioma de apresentação dos estudos nos respectivos aplicativos. Essa característica permite supor que territórios falantes de outras línguas, a exemplo do brasileiro, apesar de possuírem produções tecnológicas nesse sentido, pouco têm produzido em termos de certificação científica, o que representa deficiência, haja vista que essa etapa, no desenvolvimento de ferramenta tecnológica, especialmente no contexto da saúde, configura-se como atestado de confiança para quem o emprega.

Por conseguinte, em análise aos métodos empregados pelos pesquisadores, nos estudos incluídos, para análise do desempenho das ferramentas, considerando que a nomenclatura "evidência científica" diz respeito a um resultado obtido mediante o seguimento de etapas redutoras de vieses (Barbosa, 2010), percebe-se que a metodologia aplicada, em maioria enquadrada como Nível I, denota alto rigor avaliativo e potencial embasamento científico, o que corrobora a legitimação da utilização dos aplicativos pelo público visado dentro do setor saúde.

Ao voltar a atenção para o âmbito tecnológico, observa-se que as TIC atuam para tornar viável métodos científicos, visto que o prosseguimento dessas metodologias de forma ativa se evidencia como forma inovadora no ensino-aprendizado (Pereira et al., 2017). Assim, como as TIC, o software é desenvolvido por pessoas com competências profissionais que abrangem várias áreas e é interdependente, sendo recurso virtual que funciona a partir da interpretação de máquinas computáveis e dos seres humanos (Fernandes, 2003).

Percebe-se também que os softwares utilizados na saúde, como os aplicativos, auxiliam na avaliação do estado de saúde, incentivam hábitos saudáveis e ajudam na gestão da saúde, além de outras aplicações (Rocha et al., 2016). Por isso, tendo em vista a quantidade progressiva de aplicativos mHealth, a preocupação quanto ao uso dessas ferramentas está relacionada à necessidade de avaliação da usabilidade e das aplicações, para que sejam relevantes e tragam informações atualizadas, bem como a regulação de possíveis problemas a partir de evidências encontradas, visto que o método de validação empregada na certificação de uma tecnologia necessita estabelecer a confiança de que é adequado à finalidade (Sommerville, 2011), e que a gestão de qualidade, para ser efetiva, deverá criar um produto de valor significativo para quem produziu e os usuários (Pressman \& Maxim, 2016).

Os estudos aqui avaliados denotaram prevalência dos profissionais de saúde como público-alvo, sendo notável a relevância atual dos aplicativos mHealth para essa população, uma vez que possibilitam maior agilidade, qualidade e resolutividade nas ações assistenciais (World Health Organization, 2018). Assim, as TIC têm modificado as relações profissional/paciente, melhorando o alcance das informações sobre o processo saúde/doença que favorecem o manejo apropriado dos pacientes, assim como ampliam a capacidade para estudos de base populacional e pesquisas em larga escala (Kim et al., 2014; Lee et al., 2018; Silva et al., 2019).

Nessa perspectiva, é notória a importância desses aplicativos se fundamentarem em evidências científicas, assim como de seguirem com rigor metodológico o processo de validação que objetiva averiguar a qualidade dessas tecnologias e a respectiva eficiência (Yen \& Bakken, 2012). 
Quanto ao método utilizado nos estudos, percebe-se prevalência do processo de validação por comparação com método previamente validado, com diferentes formas de análises, que se mostrou eficaz, evidenciando a usabilidade e qualidade dessas tecnologias (Bastawrous et al., 2015; Kaciroti et al., 2020; Purves et al., 2019), de modo que, ao comparar uma mHealth em contraste a outros métodos, mostrou-se a preferência pelo uso da tecnologia pela população (Ahmed et al., 2017). Destarte, evidencia-se também o processo de validação por meio da Escala de Usabilidade do Sistema que traz protagonismo ao usuário no processo de validação de modo relevante, como evidenciado (Seeber et al., 2017).

Por fim, os dados expostos e analisados neste trabalho representam pequena amostra das produções que englobam a temática da validação de apps desenvolvidos no âmbito da saúde, cujas características não assumem o papel de regra, tendo em vista que os estudos selecionados foram filtrados por nível de evidência científica, entrando apenas os de classificação superior a III. Neste sentido, sugere-se a realização de novas pesquisas, neste perfil, contemplando amostra mais ampla do cenário tratado. Enfatiza-se, porém, que a escolha por níveis superiores de evidência científica se deu por serem estudos de resultados mais fidedignos e que, a despeito desta limitação, esta pesquisa alcançou, de maneira sólida e exitosa, o objetivo a que se propôs.

\section{Conclusão}

A avaliação de aplicativos móveis, por meio da validação, garante mais segurança à tecnologia, demonstrando eficácia, aspecto importante no que diz respeito a instrumentos que envolvam a saúde humana.

Este estudo objetivou identificar, na literatura disponível, os principais métodos adotados por pesquisadores para validação de aplicativos na área da saúde. Destarte, evidenciou-se a prevalência do processo de validação por comparação com método previamente validado, assim como por meio da Escala de Usabilidade do Sistema, demonstrando a relevância da validação de aplicativos por intermédio de métodos confiáveis e eficientes.

Portanto, enfatiza-se que a avaliação desses aplicativos beneficia profissionais da área da saúde, assim como a população. Com isso, destaca-se a necessidade do processo de validação com rigor metodológico, assim como publicações acerca dessa temática voltadas ao âmbito da saúde.

\section{Agradecimentos}

A Fundação Cearense de Apoio ao Desenvolvimento Científico e Tecnológico (FUNCAP) pelo apoio financeiro ao Programa de Bolsas de Produtividade em Pesquisa, Estímulo à Interiorização e Inovação Tecnológica - BPI

\section{Referências}

Ahmed, M., Mandic, I., Lou, W., Goodman, L., Jacobs, I., \& L'Abbé, M. (2017). Validation of a Tablet Application for Assessing Dietary Intakes Compared with the Measured Food Intake/Food Waste Method in Military Personnel Consuming Field Rations. Nutrients, 9(3). https://doi.org/10.3390/nu9030200

Araújo, W. C. O. (2020). Recuperação da informação em saúde. ConCI: Convergências em Ciência da Informação, 3(2). https://doi.org/10.33467/conci.v3i2.13447

Baig, M. M., GholamHosseini, H., Moqeem, A. A., Mirza, F., \& Lindén, M. (2019). Clinical decision support systems in hospital care using ubiquitous devices: Current issues and challenges. Health Informatics Journal, 25(3). https://doi.org/10.1177/1460458217740722

Barbosa, D. (2010). Importância da pesquisa clínica para a prática na área de saúde. Acta Paulista de Enfermagem, 23(1). https://doi.org/10.1590/S010321002010000100001

Bastawrous, A., Rono, H. K., Livingstone, I. A. T., Weiss, H. A., Jordan, S., Kuper, H., \& Burton, M. J. (2015). Development and Validation of a SmartphoneBased Visual Acuity Test (Peek Acuity) for Clinical Practice and Community-Based Fieldwork. JAMA Ophthalmology, 133(8). https://doi.org/10.1001/jamaophthalmol.2015.1468

Fernandes, J. H. C. (2003). Qual a prática do desenvolvimento de software? Ciência e Cultura, 55(2), 29-33. http://cienciaecultura.bvs.br/scielo.php?script=sci_arttext\&pid=S0009-67252003000200021\&lng=en\&nrm=iso 
Hopia, H., Latvala, E., \& Liimatainen, L. (2016). Reviewing the methodology of an integrative review. Scandinavian Journal of Caring Sciences, 30(4). https://doi.org/10.1111/scs.12327

Instituto Brasileiro de Geografia e Estatística, Diretoria de Pesquisas, Coordenação de Trabalho e Rendimento. (2017). Pesquisa Nacional por Amostra de Domicílios Contínua 2017-2018 (PNAD). IBGE.

International Data Corporation. (2021). Worldwide quarterly mobile phone tracker 2020-2025 forecast and analysis. http://www.idc.com/

Kaciroti, N., Santos, M. F., Moura, B., Bellile, E. L., Nascimento, T. D., Maslowski, E., Danciu, T. E., Donnell, A., \& Silva, A. F. (2020). SensoryDiscriminative Three-Dimensional Body Pain Mobile App Measures Versus Traditional Pain Measurement with a Visual Analog Scale: Validation Study. JMIR MHealth and UHealth, 8(8). https://doi.org/10.2196/17754

Kim, J. H., Kwon, S.-S., Shim, S. R., Sun, H. Y., Ko, Y. M., Chun, D.-I., Yang, W. J., \& Song, Y. S. (2014). Validation and Reliability of a Smartphone Application for the International Prostate Symptom Score Questionnaire: A Randomized Repeated Measures Crossover Study. Journal of Medical Internet Research, 16(2). https://doi.org/10.2196/jmir.3042

Lee, J. H., Park, Y. R., Kweon, S., Kim, S., Ji, W., \& Choi, C.-M. (2018). A Cardiopulmonary Monitoring System for Patient Transport Within Hospitals Using Mobile Internet of Things Technology: Observational Validation Study. JMIR MHealth and UHealth, 6(11). https://doi.org/10.2196/12048

Melnyk, B. M., \& Fineout-Overholt, E. (2015). Evidence-based practice in nursing \& healthcare: a guide to best practice. (3rd.). Wolters Kluwer/Lippincott Williams \& Wilkins.

Oliveira, L. B., Vilhena, B. J., Freitas, R. N., Bastos, Z. R. G., Teixeira, E., Menezes, E. G., Diniz, C. X., \& Sicsú, A. N. (2020). Aplicativos Móveis no Cuidado em Saúde: Uma Revisão Integrativa. Revista Enfermagem Atual In Derme, 93(31). https://doi.org/10.31011/reaid-2020-v.93-n.31-art.760

Page, M. J., McKenzie, J. E., Bossuyt, P. M., Boutron, I., Hoffmann, T. C., Mulrow, C. D., Shamseer, L., Tetzlaff, J. M., Akl, E. A., Brennan, S. E., Chou, R., Glanville, J., Grimshaw, J. M., Hróbjartsson, A., Lalu, M. M., Li, T., Loder, E. W., Mayo-Wilson, E., McDonald, S., ... Moher, D. (2021). The PRISMA 2020 statement: an updated guideline for reporting systematic reviews. BMJ. https://doi.org/10.1136/bmj.n71

Pereira, F. G. F., Frota, N. M., Silva, D. V., Sousa, L. M. O., Almeida, J. C., \& Cysne Filho, F. M. S. (2017). Evaluation of an application program for the teaching of vital signs. REME: Revista Mineira de Enfermagem, 21. https://doi.org/10.5935/1415-2762.20170044

Pressman, R. S., \& Maxim, B. R. (2016). Engenharia de software uma abordagem profissional (8th ed.). AMGH.

Purves, K. L., Constantinou, E., McGregor, T., Lester, K. J., Barry, T. J., Treanor, M., Sun, M., Margraf, J., Craske, M. G., Breen, G., \& Eley, T. C. (2019). Validating the use of a smartphone app for remote administration of a fear conditioning paradigm. Behaviour Research and Therapy, 123 . https://doi.org/10.1016/j.brat.2019.103475

Rocha, T. A. H., Thumé, E., Silva, N. C., Barbosa, A. C. Q., Carmo, M., Rodrigues, J. M., \& Fachini, L. A. (2016). Saúde Móvel: novas perspectivas para a oferta de serviços em saúde. Epidemiologia e Serviços de Saúde, 25(1). https://doi.org/10.5123/S1679-49742016000100016

Seeber, L., Conrad, T., Hoppe, C., Obermeier, P., Chen, X., Karsch, K., Muehlhans, S., Tief, F., Boettcher, S., Diedrich, S., Schweiger, B., \& Rath, B. (2017). Educating parents about the vaccination status of their children: A user-centered mobile application. Preventive Medicine Reports, 5. https://doi.org/10.1016/j.pmedr.2017.01.002

Silva, R. M., Brasil, C. C. P., Bezerra, I. C., \& Queiroz, F. F. S. N. (2019). Mobile health technology for gestational care: evaluation of the GestAção's app. Revista Brasileira de Enfermagem, 72(suppl 3). https://doi.org/10.1590/0034-7167-2018-0641

Sommerville, I. (2011). Engenharia de Software. (9th ed.) Pearson Education.

Souza, M. T., Silva, M. D., \& Carvalho, R. (2010). Integrative review: what is it? How to do it? Einstein (São Paulo), 8(1). https://doi.org/10.1590/s167945082010rw1134

Tibes, C. M., Dias, J. D., Westin, U. M., Domingues, A. N., Zem-Mascarenhas, S. H., \& Évora, Y. D. M. (2017). Development of digital educational resources for nursing education. Journal of Nursing UFPE on Line, 11(Supl. 3), 1326-1334. https://periodicos.ufpe.br/revist as/revistaenfermagem/article/download/13972/16816

Veiga, J., Rodriguez, J. P., Trevizan, B., Rebonatto, M. T., \& Marchi, A. C. B. (2017). Aplicações móveis com interação médico-paciente para um estilo de vida saudável: uma revisão sistemática. Revista Eletrônica de Comunicação, Informação e Inovação em Saúde, 11(1). https://doi.org/10.29397/reciis.v11i1.1188

World Health Organization. (2018). Digital health. World Health Organization. https://apps.who.int/gb/ebwha/pdf_files/WHA71/A71_R7-en.pdf?ua=1

Yen, P.-Y., \& Bakken, S. (2012). Review of health information technology usability study methodologies. Journal of the American Medical Informatics Association, 19(3). https://doi.org/10.1136/amiajnl-2010-000020 